\title{
Associations With Definitive Outcomes and Clinical Benefit of Cancer Drugs at the Time of Marketing Approval and in the Postmarketing Period
}

\author{
Aida Bujosa, $\mathrm{MD}^{1}$; Consolación Moltó, $\mathrm{MD}^{1}$; Thomas J. Hwang, $\mathrm{AB}^{2}$; José Carlos Tapia, $\mathrm{MD}^{1}$; \\ Kerstin N. Vokinger, $\mathrm{MD}^{3}$; Arnoud J. Templeton, $\mathrm{MD}^{4}$; Ignasi Gich, MD, $\mathrm{PhD}^{5}$; Agustí Barnadas, MD, PhD ${ }^{1}$; \\ Eitan Amir, $\mathrm{MD}, \mathrm{PhD}^{6}$; and Ariadna Tibau, $\mathrm{MD}, \mathrm{PhD}^{1}$
}

\begin{abstract}
Background: Most anticancer drugs are approved by regulatory agencies based on surrogate measures. This article explores the variables associated with overall survival (OS), quality of life (QoL), and substantial clinical benefit among anticancer drugs at the time of approval and in the postmarketing period. Methods: Anticancer drugs approved by the FDA between January 2006 and December 2015 and with postmarketing follow-up until April 2019 were identified. We evaluated trial-level data supporting approval and any updated OS and/or QoL data. We applied the ESMOMagnitude of Clinical Benefit Scale (ESMO-MCBS) and the ASCO Value Framework (ASCO-VF) to initial and follow-up studies. Results: We found that 58 drugs were approved for 96 indications based on 96 trials. At registration, approval was based on improved OS in 39 trials (41\%) and improved QoL in 16 of 45 indications (36\%). Postmarketing data showed an improvement in OS for 28 of 59 trials (47\%) and in QoL for 22 of 48 indications (46\%). At the time of approval, 25 of 94 (27\%) and 26 of 80 scorable trials (33\%) met substantial benefit thresholds using the ESMO-MCBS and ASCO-VF, respectively. In the postmarketing period, 37 of $69(54 \%)$ and 35 of $65(54 \%)$ trials met the substantial benefit thresholds. Drugs with companion diagnostics and immune checkpoint inhibitors were associated significantly with substantial clinical benefit. Conclusions: Compared with the time of approval, more anticancer drugs showed improved OS and QoL and met the ESMO-MCBS or ASCO-VF thresholds for substantial benefit over the course of postmarketing time. However, only approximately half of the trials met the threshold for substantial benefit. Companion diagnostic drugs and immunotherapy seemed to be associated with greater clinical benefit.
\end{abstract}

J Natl Compr Canc Netw, doi: 10.6004/jnccn.2021.7003 Published online September 24, 2021

See JNCCN.org for supplemental online content.

\section{Background}

The FDA defines clinical benefit as an improvement in either the duration or the quality of life (QoL). ${ }^{1} \mathrm{FDA}$ criteria allow cancer drugs to be approved based on surrogate measures that will reasonably predict definitive outcomes, such as overall survival (OS) or QoL. Most intermediate endpoints have not been validated as surrogates for definitive outcomes yet are used as primary endpoints in trials supporting drug approval. ${ }^{2,3}$ This practice can limit the understanding of the clinical benefit of new drugs. ${ }^{4,5}$

Recent systematic reviews have indicated that many surrogate endpoints used for drug approvals do not translate into improvements in $\mathrm{OS}^{6}$ or QoL. ${ }^{7,8}$ In addition, recent findings have revealed that fewer than half of cancer drug indications approved by the $\mathrm{FDA}^{2,3}$ and the European Medicines Agency ${ }^{9}$ have shown improved OS or QoL in the postmarketing period. Similarly, only a proportion of studies supporting drug approval have shown substantial clinical benefit using the ESMO-Magnitude of Clinical Benefit Scale (ESMOMCBS) or the ASCO Value Framework (ASCO-VF) at the time of drug approval. ${ }^{10-12}$ There are limited data on whether the magnitude of clinical benefit changes between the time of regulatory approval and the postmarketing period. In this article, we quantify the proportion of studies meeting thresholds for substantial

\footnotetext{
${ }^{1}$ Oncology Department, Hospital de la Santa Creu i Sant Pau, Institut d'Investigació Biomèdica Sant Pau, and Departament de Medicina de la Universitat Autònoma de Barcelona, Barcelona, Catalonia, Spain; ${ }^{2}$ Program on Regulation, Therapeutics, and Law, Brigham and Women's Hospital and Harvard Medical School, Boston, Massachusetts; ${ }^{3}$ Institute for Primary Care and Health Outcomes Research, University of Zürich, Zürich, Switzerland; ${ }^{4}$ Department of Medical Oncology, St. Claraspital Basel, and Faculty of Medicine, University of Basel, Basel, Switzerland; ${ }^{5}$ Department of Epidemiology, Hospital de la Santa Creu i Sant Pau, and Universitat Autònoma de Barcelona, Barcelona, Catalonia, Spain; and ${ }^{6}$ Division of Medical Oncology and Hematology, Department of Medicine, Princess Margaret Cancer Centre, and the University of Toronto, Toronto, Ontario, Canada.
} 
clinical benefit both at the time of approval and in the postmarketing period and explore associations with improved OS, QoL, and clinical benefit over time.

\section{Methods}

\section{Data Sources}

We examined data from pivotal trials supporting FDA approval in adult solid tumors between January 1, 2006, and December 31, 2015. These dates were selected to allow at least 3 years of postmarketing follow-up for all drugs. Details of data sources have been described previously. ${ }^{13}$

\section{Data Extraction, Search Strategies, and Data Analysis}

Two authors (A. Bujosa, A. Tibau) extracted data using predesigned electronic forms. For each indication, the review $^{14-17}$ and approval (accelerated vs regular) ${ }^{18}$ pathways and the number of trials supporting the application were identified. When $>1$ pivotal study supported a single indication, a hierarchical selection strategy preferring more definitive endpoints (typically OS) to QoL was used. Then, the ClinicalTrials.gov identification number was collected from the reports of registration trials. To determine changes in the postmarketing period, PubMed was searched first. Second, search results were crossreferenced with ClinicalTrials.gov, and if no follow-up trials were identified, then Google Scholar was searched.

In the postmarketing period, for regular approvals, updates of pivotal trials were analyzed as follow-up studies. For accelerated approvals, we assessed the status of conversion to full approval. ${ }^{18}$ If conversion to regular approval was granted, then only the confirmatory trial was analyzed as a follow-up study.

For each included trial, we extracted information on trial characteristics, primary efficacy endpoints (OS vs intermediate endpoints), toxicity, and QoL outcomes. We also collected data on whether a companion diagnostic test was available as defined by the FDA. ${ }^{19}$ For trials performed in the palliative setting, we collected data on the line of therapy. For postmarketing trials, we collected the median time of follow-up, the most rigorous endpoint that was statistically significant, and updated information about efficacy, QoL, and toxicity when available. For accelerated approvals converted to regular approvals, we considered the period between accelerated approval and regular approval as the median time between accelerated approval and conversion to full approval.

A drug was considered to have shown an OS benefit if a statistically significant benefit was observed with experimental therapy. ${ }^{3}$ A drug was considered to have shown a QoL benefit if a statistically significant difference was reported between the drug and the experimental arm among randomized controlled trials and between baseline and after treatment of single-arm trials. QoL data needed to be based on a global score, a subscale, or a specific item from a validated patient-reported outcome (PRO) instrument. $^{3}$

\section{Data Scoring}

We used the ASCO-VF Net Health Benefit Score version $2.0^{20}$ and the ESMO-MCBS version $1.1^{21}$ to quantify the magnitude of clinical benefit. Despite differences in how efficacy, toxicity, and QoL are weighed (the ASCO-VF is linear and the ESMO-MCBS is categorical), both frameworks aim to measure the magnitude of the benefit of novel therapies. ESMO-MCBS and ASCO-VF grades were applied independently by 2 authors (either A. Bujosa and J.C. Tapia, or A. Bujosa and C. Moltó, respectively). The discrepancies were resolved by a fourth author (A. Tibau). In the postmarketing period, for drugs with updated efficacy and QoL data, grades and/or scores were recalculated because updated efficacy or QoL data can change scores. For trials in which updated data reported only 1 domain of efficacy or QoL, grades and/or scores were adjusted, retaining initial data that were not updated. Substantial clinical benefit was defined as follows: for the ASCO-VF, a threshold score of $\geq 45,{ }^{22}$ and for the ESMO-MCBS, a grade of A or B for trials of curative intent and 4 or 5 for those of palliative intent. ${ }^{21}$

\section{Statistical Analysis}

Data were reported descriptively as proportions, medians, and ranges. In the noncurative setting, we explored whether characteristics of trials and applications were associated with OS, QoL, and clinically significant benefit thresholds. Associations were evaluated using univariable logistic regression at 3 timepoints: (1) at the time of approval, (2) after maximum postmarketing time, and (3) at any point in the life cycle of the drug. Regression results were reported as odds ratios and their respective $95 \%$ confidence intervals. Multivariable models included variables that were statistically significant in univariable analysis, with variable selection ensuring adequate fitting of the model. To reduce the chance of overfitting, multivariable analysis was performed exclusively using data at any point in the life cycle of the drug. Such analyses were not planned in the curative setting because of the small number of studies supporting such indications. Trends over time were assessed using the chi-square test for trends. All analyses were conducted using SPSS Statistics, version 21 (IBM Corp). Statistical tests were 2-sided, and statistical significance was defined as a 2 -tailed $P<.05$.

\section{Results}

Between January 1, 2006, and December 31, 2015, 58 drugs were approved for 96 solid tumor indications based on 96 trials. Characteristics of included applications and trials supporting drug approval at the time of market authorization 


\begin{tabular}{|c|c|}
\hline Characteristics & n (\%) \\
\hline \multicolumn{2}{|l|}{ Application characteristics } \\
\hline Indications available & $96(100)$ \\
\hline \multicolumn{2}{|l|}{ Priority review designation } \\
\hline Yes & $72(75)$ \\
\hline No & $24(25)$ \\
\hline \multicolumn{2}{|c|}{ Breakthrough therapy designation $^{a}$} \\
\hline Yes & $9 / 50(18)$ \\
\hline No & $41 / 50(82)$ \\
\hline \multicolumn{2}{|l|}{ Orphan drug designation } \\
\hline Yes & $44(46)$ \\
\hline No & $52(54)$ \\
\hline \multicolumn{2}{|l|}{ Type of approval } \\
\hline Regular & $74(77)$ \\
\hline Accelerated & $22(23)$ \\
\hline \multicolumn{2}{|l|}{ Type of marketing authorization } \\
\hline Initial & $45(47)$ \\
\hline Supplemental & $51(53)$ \\
\hline \multicolumn{2}{|c|}{ Number of trials supporting approval indication } \\
\hline Multiple & $13(14)$ \\
\hline Single & $83(86)$ \\
\hline \multicolumn{2}{|l|}{ Trial characteristics } \\
\hline Studies available & $96(100)$ \\
\hline \multicolumn{2}{|l|}{ Study design } \\
\hline Randomized controlled trial & $82(85)$ \\
\hline Single-arm trial & $14(15)$ \\
\hline \multicolumn{2}{|l|}{ Phase of study } \\
\hline I-II & $18(19)$ \\
\hline III & $78(81)$ \\
\hline \multicolumn{2}{|l|}{ Blinding } \\
\hline Open-label & $59(62)$ \\
\hline Double-blind & $37(38)$ \\
\hline
\end{tabular}

are shown in Table 1. Of these indications, 74 (77\%) were granted regular approval and $22(23 \%)$ were granted accelerated approval. Fourteen approvals (15\%) were supported by single-arm studies. For 88 indications (92\%), treatment intent was noncurative.

In the postmarketing period, most accelerated approval indications were converted to regular approval. Conversion from accelerated to regular approval was supported by 20 trials. One indication was pending regular approval (nivolumab and ipilimumab for advanced melanoma), and 1 indication was withdrawn (bevacizumab for advanced breast cancer). Consequently, the analysis comprised 96 trials: 74 trials supporting regular approval, 20

\begin{tabular}{|c|c|}
\hline Characteristics & n (\%) \\
\hline \multicolumn{2}{|l|}{ Trial characteristics (cont.) } \\
\hline \multicolumn{2}{|l|}{ Intent of treatment } \\
\hline Curative & $8(8)$ \\
\hline Noncurative & $88(92)$ \\
\hline \multicolumn{2}{|l|}{ Line of treatment ${ }^{b}$} \\
\hline First line & $34 / 88(39)$ \\
\hline Later lines & $54 / 88(61)$ \\
\hline \multicolumn{2}{|l|}{ Type of solid tumor } \\
\hline Lung, breast, colorectal, prostate & $43(45)$ \\
\hline Others & $53(55)$ \\
\hline \multicolumn{2}{|l|}{ Experimental drug type } \\
\hline Chemotherapy & $16(17)$ \\
\hline Hormonal therapy & $3(3)$ \\
\hline Target therapy & $66(68)$ \\
\hline Immunotherapy & $11(12)$ \\
\hline \multicolumn{2}{|l|}{ Companion diagnostic $^{c}$} \\
\hline Yes & $21(22)$ \\
\hline No & $75(78)$ \\
\hline \multicolumn{2}{|l|}{ Basis of decision } \\
\hline Subgroup analysis & $7(7)$ \\
\hline Entire study population & 89 (93) \\
\hline \multicolumn{2}{|l|}{ Crossover $^{d}$} \\
\hline Yes & $26 / 82(31)$ \\
\hline No & $53 / 82(65)$ \\
\hline Unknown & $3 / 82(4)$ \\
\hline
\end{tabular}

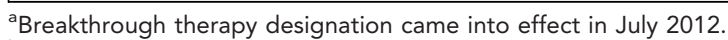

${ }^{\mathrm{b}}$ Trials with curative intent were not considered.

${ }^{\circ}$ Classification of a drug as having a companion diagnostic test was determined by the FDA. ${ }^{19}$

dOnly 82 randomized controlled trials were considered.

postmarketing trials supporting conversion to regular approval, 1 trial supporting the FDA withdrawal of the breast cancer indication for bevacizumab, and 1 trial supporting accelerated approval and pending conversion to regular approval.

\section{OS and QoL Data}

Table 2 summarizes the results for OS, QoL, and intermediate endpoints for all included studies. With a median postmarketing period of 3.3 years (minimum 9.2 months, maximum 8.8 years), 70 trials $(73 \%)$ reported updated data on efficacy $(n=59 ; 61 \%)$ and/or QoL $(n=48 ; 50 \%)$. For 26 trials $(27 \%)$, no updated data on efficacy or QoL were available.

At the time of marketing, approval was based on OS in 39 trials $(41 \%)$. Among the 59 trials providing updated efficacy data in the postmarketing setting, 28 (47\%) showed 
Table 2. OS, QoL, and Clinical Benefit for Trials Included in Original FDA Report and in Postmarketing Period

\begin{tabular}{|c|c|c|c|}
\hline Original FDA Report & $\mathbf{n}$ & Updated Report & $\mathbf{n}$ \\
\hline Effect on efficacy & & Effect on efficacy & \\
\hline Confirmed & 96 & Updated & 59 \\
\hline OS benefit ${ }^{a}$ & 39 (41\%) & OS benefit ${ }^{\mathrm{a}}$ & $28(47 \%)$ \\
\hline Surrogate endpoint & $57(59 \%)$ & Different from initial report & 11 \\
\hline PFS & 35 & Same as initial report & 17 \\
\hline DFS & 1 & Surrogate endpoint & $31(53 \%)$ \\
\hline ORR & 20 & Different from initial report & 8 \\
\hline Biochemical & 1 & Same as initial report & 23 \\
\hline Unknown & 0 & Unknown & 37 \\
\hline Effect on QoL & & Effect on QoL & \\
\hline Confirmed & 45 & Updated & 48 \\
\hline Benefit $^{\mathrm{b}}$ & $16(36 \%)$ & Benefit $^{b}$ & $22(46 \%)$ \\
\hline No benefit & 29 (64\%) & No benefit & $26(54 \%)$ \\
\hline Unknown & 51 & Unknown & 48 \\
\hline ASCO-VF clinical benefit & & ASCO-VF clinical benefit & \\
\hline Evaluable & 80 & Evaluable & 65 \\
\hline High benefit ${ }^{c}$ & $26(33 \%)$ & High benefit $^{c}$ & $35(54 \%)$ \\
\hline Low benefit ${ }^{d}$ & $54(67 \%)$ & Low benefit ${ }^{d}$ & $30(46 \%)$ \\
\hline Not evaluable & 16 & Not evaluable & 31 \\
\hline ESMO-MCBS clinical benefit & & ESMO-MCBS clinical benefit & \\
\hline Evaluable & 94 & Evaluable & 69 \\
\hline High benefit ${ }^{\mathrm{e}}$ & $25(27 \%)$ & High benefit ${ }^{\mathrm{e}}$ & $37(54 \%)$ \\
\hline Low benefit ${ }^{\dagger}$ & $69(73 \%)$ & Low benefit ${ }^{f}$ & $32(46 \%)$ \\
\hline Not evaluable & 2 & Not evaluable & 27 \\
\hline
\end{tabular}

Abbreviations: ASCO-VF, ASCO Value Framework; DFS, disease-free survival; ESMO-MCBS, ESMO-Magnitude of Clinical Benefit Scale; ORR, overall response rate; OS, overall survival; PFS, progression-free survival; QoL, quality of life.

${ }^{a}$ A drug was considered to have shown an OS benefit if a statistically significant benefit was observed with experimental therapy.

${ }^{b}$ A drug was considered to have shown a QoL benefit if a statistically significant difference was reported between the drug and experimental arm among randomized controlled trials and between baseline and after treatment in single-arm trials.

"High clinical benefit for ASCO-VF was considered a threshold score of $\geq 45$ (continuous variable).

dLow clinical benefit for ASCO-VF was considered a score of $<44$ points.

e High clinical benefit for ESMO-MCBS was considered a grade of A or B for trials of curative intent and a grade of 4 or 5 for those of palliative intent.

fLow clinical benefit for ESMO-MCBS was considered a grade of $C$ for trials of curative intent and grade 1 to 3 for those of palliative intent.

improvement in OS, including 11 (19\%) that showed OS improvement for the first time in the postmarketing period and 17 (29\%) in which OS benefit reported previously was confirmed. In total, significantly improved OS was observed in 50 of 96 indications (52\%), no improvement was observed in 31 indications (32\%), and for 15 indications (16\%), the effect on OS remains unknown.

Only 45 of 96 (47\%) initial indications reported QoL data. Of these, 16 indications (36\%) showed a statistically significant improvement in at least $1 \mathrm{PRO}$ at the time of market approval. In the postmarketing period, among the 48 trials (50\%) reporting updated QoL data, an improvement in QoL was observed for 22 (46\%): 11 drugs
(23\%) showed improvement in QoL for the first time, and in 11 trials $(23 \%)$ a QoL benefit reported previously was confirmed. Overall, a significant improvement in QoL was observed for 27 of 96 indications (28\%), and there was no improvement for 36 indications (36\%). For 33 indications (34\%), QoL was not assessed or reported formally. Notably, 16 indications (17\%) showed an improvement in both OS and QoL.

\section{Measures of Substantial Clinical Benefit}

Table 2 shows the ASCO-VF and the ESMO-MCBS scores at the time of approval and in the postmarketing period. ASCO-VF scores were applied to 80 of 96 initial trials 
(83\%). Of the trials for which ASCO-VF could not be applied, 14 were single-arm or the primary endpoint was not suitable for assessment. Only 26 trials (33\%) evaluable by the ASCO-VF met the threshold for substantial clinical benefit at the time of marketing approval. In the postmarketing period, 65 trials $(68 \%)$ provided updated information and were evaluable using the ASCO-VF. Of these, 35 trials (54\%) met the ASCO-VF scores for substantial clinical benefit including $17(26 \%)$ that did not initially meet the threshold for substantial benefit but were upgraded to show substantial clinical benefit based on postmarketing data. Four trials $(6 \%)$ that showed substantial benefit at the time of approval were downgraded because of a lack of substantial benefit based on postmarketing data.

The ESMO-MCBS scores could be applied to 94 of 96 initial trials (98\%). Only 25 trials (27\%) met the ESMOMCBS threshold for substantial clinical benefit at the time of marketing approval. In the postmarketing period, 69 trials provided updated information and were scorable for the ESMO-MCBS. Of these, 37 (54\%) met the threshold for substantial clinical benefit. Updated results led to a change in the ESMO-MCBS substantial clinical benefit threshold in 18 trials (26\%). Of these, 17 trials $(25 \%)$ that did not initially meet the threshold for substantial benefit were upgraded to show substantial clinical benefit based on postmarketing data. One trial $(1 \%)$ that showed substantial benefit at the time of approval was downgraded because of a lack of substantial benefit based on postmarketing data.

Overall, based on both initial and updated data, among the 96 indications approved by the FDA, 39 trials (41\%) met the criteria for substantial benefit using the ASCO-VF and 42 (44\%) met the threshold using the ESMO-MCBS.

Over time, there has been an increase in the number of trials meeting the ASCO-VF threshold at any time (11\% for drugs approved in 2006 vs $56 \%$ for drugs approved in 2015 ; $P_{\text {trend }}=.038$ ). There were no significant changes over time in OS or QoL or in the ESMO-MCBS scores. An OS benefit was observed among $60 \%$ of drugs approved in 2006 and $55 \%$ approved in 2015 ( $P_{\text {trend }}=.78$ ). A QoL benefit was observed in $17 \%$ of drugs approved in 2006 and $54 \%$ approved in 2015 ( $P_{\text {trend }}=.17$ ), and for the ESMO-MCBS, $40 \%$ of drugs met the substantial clinical benefit threshold in 2006 and $47 \%$ in 2015 ( $P_{\text {trend }}=.65$ ).

\section{Associations With OS, QoL, and Clinical Benefit}

Associations with OS, QoL, and magnitude of clinical benefit as measured using the ASCO-VF and ESMO-MCBS at the time of market authorization are shown in Table 3 and those in the postmarketing period are shown in Table 4.

At the time of market approval, there were statistically significant associations between improved OS and regular approval, nonorphan drug designation, sample size, more common tumors compared with others, and absence of crossover interference. Factors associated with OS in the postmarketing period differed, with immunotherapy, companion diagnostic drugs, and approvals based on subgroup analysis showing a survival advantage.

For QoL, there were no significant associations at initial approval. Improved QoL in the postmarketing period was associated with sample size, immunotherapy, and open-label studies.

At the time of market authorization, initial indications and those reporting QoL benefit were more likely to show substantial clinical benefit as determined by the ASCO-VF. In the postmarketing period, substantial clinical benefit as measured by the ASCO-VF was associated with accelerated approvals, drugs granted breakthrough therapy designation, indications for later lines of therapy, indications supported by phase I-II or single-arm trials, and indications with a lower sample size. Drugs initially approved with evidence of improved QoL were associated with substantial clinical benefit both at the time of approval and in the postmarketing period.

For the ESMO-MCBS, at the time of market authorization, drugs with a companion diagnostic test and those with OS benefit or QoL improvement were associated with substantial clinical benefit. Postapproval, only trials of drugs with companion diagnostics were associated with substantial clinical benefit.

Associations with OS, QoL, or substantial clinical benefit at any time are shown in supplemental eTable 1 (available with this article at JNCCN.org). In multivariable analyses, drugs with companion diagnostic tests and immunotherapy were associated with improved OS, QoL, and substantial clinical benefit.

\section{Discussion}

In the current analysis, we found that at the time of market approval, among 96 cancer drug indications approved by the FDA between 2006 and 2015, two-thirds of trials supporting FDA-approved anticancer drugs failed to meet the thresholds of substantial clinical benefit established by the ASCO-VF and ESMO-MCBS. With 3.3 years of postmarketing experience, approximately half of the trials showed substantial clinical benefit, thereby increasing the number of trials meeting ASCO-VF and ESMO-MCBS thresholds. Our analysis also showed an increase in the number of trials meeting the ASCO-VF threshold for clinical benefit over time.

There are limited data regarding the impact of postmarketing experience on the magnitude of clinical benefit for FDA-approved anticancer drugs as measured by validated frameworks. Previous data have focused on the availability of data on OS and QoL at the time of drug approval by the FDA from 2011 to $2017^{3}$ and by the 


\begin{tabular}{|c|c|c|c|c|c|c|c|c|}
\hline & \multicolumn{2}{|c|}{ OS Benefit ${ }^{a}$} & \multicolumn{2}{|c|}{ QoL Benefit ${ }^{\mathbf{b}}$} & \multicolumn{2}{|c|}{ ASCO-VF Clinical Benefit ${ }^{c}$} & \multicolumn{2}{|c|}{ ESMO-MCBS Clinical Benefit } \\
\hline & OR $(95 \% \mathrm{Cl})$ & $P$ Value ${ }^{e}$ & OR $(95 \% \mathrm{Cl})$ & $P$ Value $^{e}$ & OR $(95 \% \mathrm{Cl})$ & $P$ Value ${ }^{e}$ & OR $(95 \% \mathrm{Cl})$ & $P$ Value ${ }^{e}$ \\
\hline $\begin{array}{l}\text { Regular approval (vs } \\
\text { accelerated approval) }\end{array}$ & $21.38(2.71-168.8)$ & .004 & $0.30(0.05-1.89)$ & .20 & $5.05(0.60-42.41)$ & .14 & $1.78(0.46-6.86)$ & .40 \\
\hline $\begin{array}{l}\text { Orphan drug designation } \\
\text { (vs not) }\end{array}$ & $0.39(0.16-0.95)$ & .04 & $1.60(0.40-6.36)$ & .50 & $0.99(0.37-2.65)$ & .98 & $1.41(0.51-3.90)$ & .51 \\
\hline $\begin{array}{l}\text { Priority review designation } \\
\text { (vs not) }\end{array}$ & $1.96(0.71-5.40)$ & .19 & $3.00(0.66-13.66)$ & .16 & $3.61(0.94-13.82)$ & .06 & - & - \\
\hline $\begin{array}{l}\text { Breakthrough therapy } \\
\text { designation }\end{array}$ & $0.29(0.05-1.56)$ & .15 & - & - & $3.00(3.68-24.50)$ & .30 & $1.61(0.33-7.78)$ & .55 \\
\hline $\begin{array}{l}\text { Initial approval } \\
\text { (vs supplemental) }\end{array}$ & $0.70(0.30-1.63)$ & .41 & $2.92(0.77-11.07)$ & .12 & $3.26(1.17-9.08)$ & .02 & $1.93(0.68-5.49)$ & .22 \\
\hline $\begin{array}{l}\text { Multiple trials supporting } \\
\text { approval (vs } 1 \text { trial) }\end{array}$ & $0.31(0.06-1.54)$ & .15 & $4.85(0.46-51.66)$ & .19 & $2.18(0.29-16.51)$ & .45 & $2.76(0.69-11.01)$ & .15 \\
\hline $\begin{array}{l}\text { Sample size per } 100 \\
\text { patients }\end{array}$ & $1.70(1.36-2.12)$ & $<.001$ & $0.98(0.82-1.18)$ & .86 & $0.91(0.77-1.06)$ & .23 & $1.04(0.90-1.20)$ & .56 \\
\hline $\begin{array}{l}\text { Lung, breast, colorectal, } \\
\text { and prostate cancer } \\
\text { (vs others) }\end{array}$ & $2.40(1.04-5.88)$ & .04 & $3.18(0.82-12.34)$ & .09 & $0.77(0.29-2.07)$ & .61 & $0.71(0.26-1.97)$ & .51 \\
\hline $\begin{array}{l}\text { Immunotherapy } \\
\text { (vs standard and } \\
\text { target therapy) }\end{array}$ & $1.12(0.28-4.47)$ & .88 & - & - & $1.64(0.34-8.00)$ & .54 & $3.36(0.80-14.04)$ & .09 \\
\hline $\begin{array}{l}\text { Companion diagnostic } \\
\text { (vs none) }\end{array}$ & $0.32(0.09-1.07)$ & .06 & $8.00(0.80-80.41)$ & .08 & $3.00(0.81-11.08)$ & .09 & $4.22(1.36-13.07)$ & .01 \\
\hline 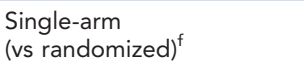 & - & - & $7.00(0.70-70.04)$ & .09 & - & - & $0.61(0.12-3.02)$ & .54 \\
\hline Phase I-II (vs phase III) ${ }^{f}$ & - & - & 9.54 (0.99-92.17) & .05 & $1.42(0.22-9.14)$ & .71 & $0.78(0.20-3.08)$ & .72 \\
\hline $\begin{array}{l}\text { Approval based on } \\
\text { subgroup analysis (vs not) }\end{array}$ & $0.53(0.10-2.87)$ & .46 & - & - & $2.18(0.29-16.51)$ & .45 & $0.57(0.06-5.08)$ & .62 \\
\hline $\begin{array}{l}\text { Open-label } \\
\text { (vs double-blind) }\end{array}$ & $0.78(0.33-1.85)$ & .57 & $3.85(0.98-15.12)$ & .05 & $1.09(0.41-2.89)$ & .86 & $2.21(0.72-6.82)$ & .17 \\
\hline Crossover (vs not) & $0.16(0.05-0.49)$ & .001 & $1.03(0.26-4.11)$ & .97 & $2.29(0.82-6.40)$ & .11 & $1.44(0.47-4.40)$ & .52 \\
\hline Later lines (vs first-line) & $1.06(0.44-2.53)$ & .90 & $1.86(0.51-6.84)$ & .35 & $2.07(0.73-5.86)$ & .17 & $1.52(0.51-4.47)$ & .45 \\
\hline $\begin{array}{l}\text { OS (vs intermediate } \\
\text { endpoints) }\end{array}$ & - & - & $0.54(0.15-1.98)$ & .35 & $0.61(0.23-1.63)$ & .32 & $2.95(1.03-8.46)$ & .04 \\
\hline QoL benefit & $0.54(0.15-1.98)$ & .35 & - & - & $7.44(1.44-38.41)$ & .02 & $29.33(3.04-282.90)$ & .003 \\
\hline
\end{tabular}

Bold indicates statistically significant $P$ value.

Abbreviations: ASCO-VF, ASCO Value Framework; ESMO-MCBS, ESMO-Magnitude of Clinical Benefit Scale; OR, odds ratio; OS, overall survival; QoL, quality of life.

${ }^{a} A$ drug was considered to have shown an OS benefit if a statistically significant benefit was observed with experimental therapy.

${ }^{\mathrm{b}} \mathrm{A}$ drug was considered to have shown a QoL benefit if a statistically significant difference was reported between the drug and experimental arm among

randomized controlled trials and between baseline and after treatment in single-arm trials.

${ }^{c} H$ High clinical benefit for ASCO-VF was considered a threshold score of $\geq 45$.

${ }^{\mathrm{d}}$ High clinical benefit for ESMO-MCBS was considered a grade of A or B for trials of curative intent and a grade of 4 or 5 for those of palliative intent.

eBased on univariable logistic regression. All $P$ values are 2-sided.

${ }^{f}$ Statistical analysis not feasible (no single-arm trials or phase I-II studies at the time of market approval showing OS benefit. Single-arm trials not scorable with the ASCO-VF.

European Medicines Agency from 2009 to 2013. ${ }^{9}$ Taken together, these studies found that less than half of the anticancer drugs showed an OS benefit or a QoL improvement. These results are consistent with our present work, which covers a longer period. Between 2006 and 2015, the FDA approved most cancer drugs without evidence of OS $(60 \%)$ or improved QoL (83\%). Even after a median of 3.3 years, only $52 \%$ of indications were supported by an improvement in OS and $28 \%$ showed a statistically significant improvement in QoL.
When we evaluated associations with clinical benefit, we found that factors associated with OS and QoL benefit or substantial clinical benefit differed at the time of approval and in the postmarketing period. Unsurprisingly, trials with an OS benefit at marketing authorization were more likely to be granted regular approval, more commonly evaluated drugs in common cancers, and had a larger magnitude of effect. In contrast, clinical benefit in the postmarketing period was associated with immunotherapy, a treatment for which responses and durability of response 


\section{Table 4. Predictors of Benefit in the Noncurative Setting in Postmarketing Period}

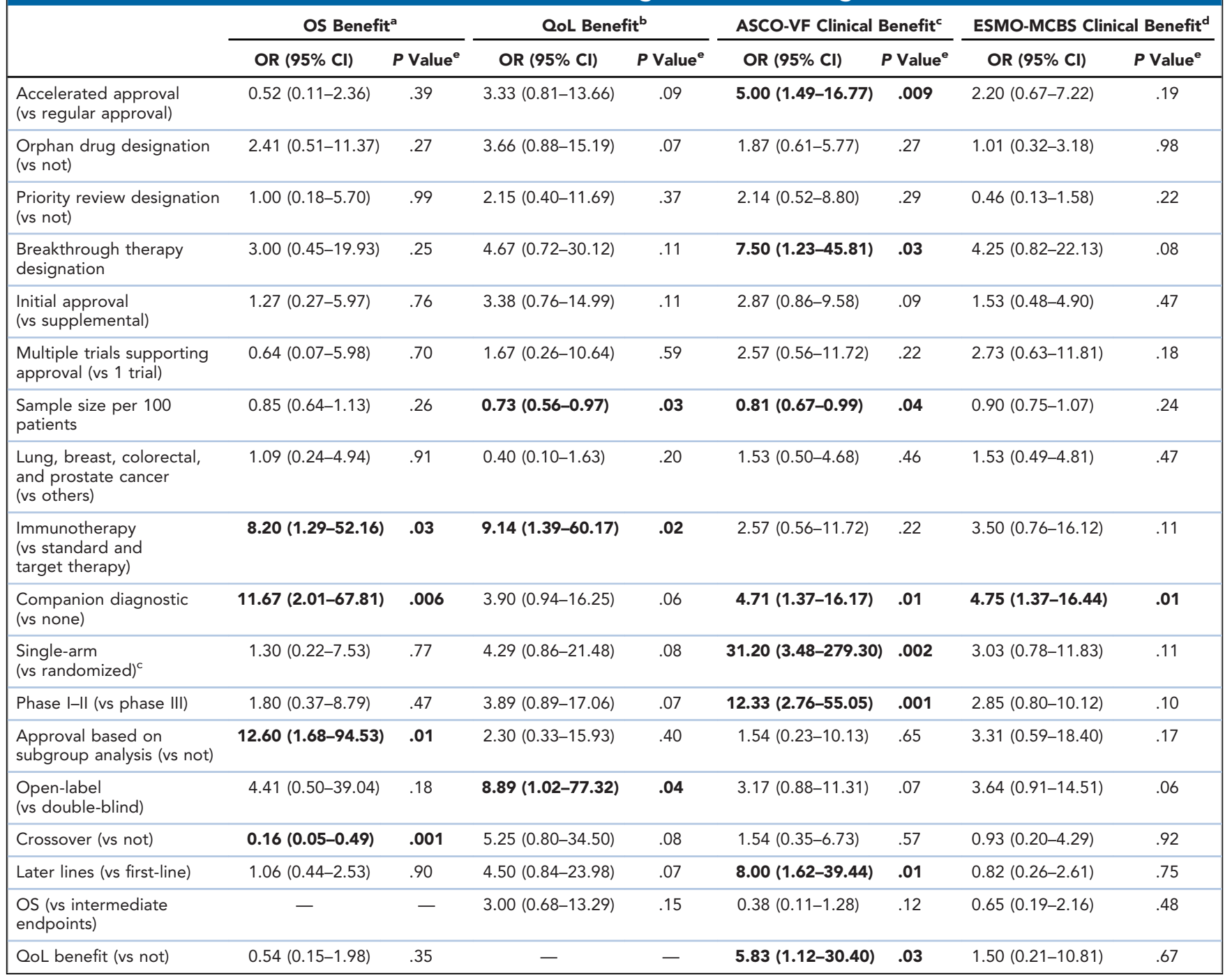

Bold indicates statistically significant $P$ value.

Abbreviations: ASCO-VF, ASCO Value Framework; ESMO-MCBS, ESMO-Magnitude of Clinical Benefit Scale; OR, odds ratio; OS, overall survival; QoL, quality of life.

${ }^{a} A$ drug was considered to have shown an OS benefit if a statistically significant benefit was observed with experimental therapy.

${ }^{\mathrm{b}} \mathrm{A}$ drug was considered to have shown a QoL benefit if a statistically significant difference was reported between the drug and experimental arm among randomized controlled trials and between baseline and after treatment in single-arm trials.

'High clinical benefit for ASCO-VF was considered a threshold score of $\geq 45$.

${ }^{\mathrm{d}}$ High clinical benefit for ESMO-MCBS was considered a grade of A or B for trials of curative intent and a grade of 4 or 5 for those of palliative intent.

Based on univariable logistic regression. All $P$ values are 2 -sided.

can appear later in follow-up. ${ }^{23}$ In addition, some variables such as drugs with companion diagnostics seemed to show a benefit in certain scenarios. Of concern was our finding that open-label studies were more likely to show improved QoL. This raises concern about ascertainment bias. $^{24}$

A possible explanation for the variability in associations of study and approval characteristics with OS, QoL, and magnitude of clinical benefit is that the ASCO-VF and ESMO-MCBS evaluate benefit as composite outcomes weighting efficacy, toxicity, and QoL rather than assessing these outcomes in isolation. Another explanation is that in our study a drug was considered to have shown an OS benefit if a statistically significant benefit was observed with experimental therapy. Statistical significance can be observed with small effect sizes, which may not meet the thresholds for substantial clinical benefit, a concept better addressed with the ASCO-VF and ESMO-MCBS, in which efficacy outcomes are measured in terms of relative and absolute benefits. 
The increased use of surrogate endpoints as the basis for approval is associated with uncertainty about the definitive benefit of drugs. This ambiguity makes postmarketing data very valuable. ${ }^{25}$ Although the accelerated approval pathway requires the completion of postmarketing clinical trials to confirm that a drug provides clinical benefit as predicted by the surrogate endpoint, regular approvals do not require further assessment to confirm effectiveness and safety after marketing authorization. Unfortunately, in the postapproval period, only two-thirds of pivotal trials provided updated data on efficacy, which may reflect a lack of positive studies (publication bias) or studies that were underpowered to show statistically significant improvement in OS.

Our data support possible improvement in the approval process for anticancer drugs. First, when surrogate endpoints are used, and especially for accelerated drug approvals, postmarketing studies with both OS and QoL outcomes should be mandated. ${ }^{26}$ Second, greater efforts need to be made to develop and validate meaningful surrogate endpoints for OS and QoL. Third, PROs and other patient-centered measures should be prioritized at early stages of drug development. In pivotal trials, missing data $^{27}$ should be minimized and journal editors should encourage the publication of such data. ${ }^{28}$ Finally, trials should be designed with sufficient statistical power for an analysis of changes in OS and/or QoL.

This study has limitations. First, the duration of postmarketing approval was relatively short. A longer period of postmarketing time may have resulted in a higher proportion of indications showing improved OS and QoL, especially with immune checkpoint inhibitors. Second, PRO data are often missing from FDA cancer-drug labels and are frequently not included in primary publications of pivotal trials. Third, PROs are often exploratory, which makes their interpretation difficult. Fourth, results obtained from analysis with frameworks that rely largely on randomized trials should be treated with caution because the score applied to experimental therapy is highly dependent on the quality of the control arm. Finally, we excluded drugs for hematologic malignancies, thereby limiting the generalizability to solid tumor drug approval.

\section{Conclusions}

In patients with solid tumors, an increasing number of approved drugs showed improved OS and QoL and met the ASCO-VF or ESMO-MCBS threshold for substantial benefit over the course of postmarketing time compared with the time of approval. However, fewer than half of the trials supporting FDA approval showed an OS benefit or a QoL improvement, and just more than half of the trials showed substantial clinical benefit. With an increasing number of drug approvals being based on single-arm trials and surrogate endpoints, ${ }^{29}$ robust postmarketing trials are becoming increasingly important. ${ }^{30}$ Regulators and professional societies should prioritize the collection of outcome data throughout the postmarketing life cycle of drugs and be prepared to update indications or treatment recommendations on the basis of all available data.

Submitted November 1, 2020; final revision received January 7, 2021 accepted for publication January 7, 2021

Published online September 24, 2021.

Previous presentation: Presented at the 2020 ASCO Annual Meeting; May 29-31, 2020. Abstract 7052. doi, 10.1200/JCO.2020.38.15_suppl.7052

Author contributions: Formal analysis: Bujosa, Gich, Tibau. Methodology: Bujosa, Molto, Tapia, Tibau. Supervision: Amir, Tibau. Writing - original draft: Bujosa, Tibau. Writing - review and editing: Molto, Hwang, Tapia, Vokinger, Templeton, Gich, Barnadas, Amir.

Disclosures: Dr. Bujosa has disclosed receiving a travel grant from Roche. Dr. Tapia has disclosed receiving travel grants from Roche and Grünenthal Group. Dr. Templeton has disclosed receiving travel grants from Janssen, Sanofi, Ipsen, and Roche, and honoraria from Astellas. Dr. Barnadas has disclosed receiving travel grants from Pfizer and Roche, and honoraria from Novartis, Pfizer, and Lilly. Dr. Amir has disclosed providing expert testimony for Genentech/Roche and serving as a consultant for Sandoz, Apobiologix, Agendia, and Myriad Genetics. Dr. Tibau has disclosed receiving a travel grant from Roche, Ipsen, Pfizer, and Lilly, and honoraria from Eisai. The remaining authors have disclosed that they have not received any financial consideration from any person or organization to support the preparation, analysis, results, or discussion of this article.

Correspondence: Ariadna Tibau, MD, PhD, Oncology Department, Hospita de la Santa Creu i Sant Pau, Institut d'Investigació Biomèdica Sant Pau, and Universitat Autònoma de Barcelona, Carrer Mas Casanovas, 90, 08041

Barcelona, Catalonia, Spain. Email: ATibau@santpau.cat

\section{References}

1. Adequate and well-controlled studies. 21 CFR $\S 314.126$ (2020).

2. Kim C, Prasad V. Cancer drugs approved on the basis of a surrogate end point and subsequent overall survival: an analysis of 5 tears of US Food and Drug Administration approvals. JAMA Intern Med 2015;175: 1992-1994.

3. Zettler M, Basch E, Nabhan C. Surrogate end points and patient-reported outcomes for novel oncology drugs approved between 2011 and 2017. JAMA Oncol 2019;5:1358-1359.

4. Tibau A, Molto C, Borrell M, et al. Magnitude of clinical benefit of cancer drugs approved by the US Food and Drug Administration based on singlearm trials. JAMA Oncol 2018;4:1610-1611.

5. Shepshelovich D, Tibau A, Goldvaser H, et al. Postmarketing modifications of drug labels for cancer drugs approved by the US Food and Drug
Administration between 2006 and 2016 with and without supporting randomized controlled trials. J Clin Oncol 2018;36:1798-1804.

6. Prasad V, Kim C, Burotto M, et al. The strength of association between surrogate end points and survival in oncology: a systematic review of triallevel meta-analyses. JAMA Intern Med 2015;175:1389-1398.

7. Kovic B, Jin X, Kennedy SA, et al. Evaluating progression-free survival as a surrogate outcome for health-related quality of life in oncology: a systematic review and quantitative analysis. JAMA Intern Med 2018;178:1586-1596.

8. Hwang TJ, Gyawali B. Association between progression-free survival and patients' quality of life in cancer clinical trials. Int J Cancer 2019;144:17461751.

9. Davis C, Naci H, Gurpinar E, et al. Availability of evidence of benefits on overall survival and quality of life of cancer drugs approved by European 
Medicines Agency: retrospective cohort study of drug approvals 2009-13. BMJ 2017;359:j4530.

10. Del Paggio JC, Sullivan R, Schrag D, et al. Delivery of meaningful cancer care: a retrospective cohort study assessing cost and benefit with the ASCO and ESMO frameworks. Lancet Oncol 2017;18:887-894.

11. Vokinger KN, Hwang TJ, Grischott T, et al. Prices and clinical benefit of cancer drugs in the USA and Europe: a cost-benefit analysis. Lancet Oncol 2020;21:664-670

12. Booth CM, Del Paggio JC. Approvals in 2016: questioning the clinical benefit of anticancer therapies. Nat Rev Clin Oncol 2017;14:135-136.

13. Tibau A, Molto C, Ocana A, et al. Magnitude of clinical benefit of cancer drugs approved by the US Food and Drug Administration. J Natl Cancer Inst 2018;110:486-492.

14. U.S. Food \& Drug Administration. New molecular entity (NME) drug and new biologic approvals. Accessed July 20, 2021. Available at: https://www. fda.gov/drugs/nda-and-bla-approvals/new-molecular-entity-nme-drugand-new-biologic-approvals

15. U.S. Food \& Drug Administration. Efficacy supplement approvals. Accessed July 20, 2021. Available at: https://www.fda.gov/drugs/nda-andbla-approvals/efficacy-supplement-approvals

16. U.S. Food \& Drug Administration. CDER breakthrough therapy designation approvals. Accessed July 20, 2021. Available at: https://www.fda.gov/ media/95302/download

17. U.S. Food \& Drug Administration. Search orphan drug designations and approvals. Accessed July 20, 2021. Available at: http://www.accessdata. fda.gov/scripts/opdlisting/oopd/index.cfm

18. U.S. Food \& Drug Administration. Accelerated approvals. Accessed July 20, 2021. Available at: https://www.fda.gov/drugs/drug-and-biologicapproval-and-ind-activity-reports/nda-and-bla-approvals

19. U.S. Food \& Drug Administration. List of cleared or approved companion diagnostic devices (in vitro and imaging tools). Accessed July 21, 2021. Available at: https://www.fda.gov/medical-devices/in-vitro-diagnostics/ list-cleared-or-approved-companion-diagnostic-devices-in-vitro-andimaging-tools
20. Schnipper LE, Davidson NE, Wollins DS, et al. Updating the American Society of Clinical Oncology Value Framework: revisions and reflections in response to comments received. J Clin Oncol 2016;34: 2925-2934.

21. Cherny NI, Dafni U, Bogaerts J, et al. ESMO-Magnitude of Clinical Benefit Scale version 1.1. Ann Oncol 2017;28:2340-2366.

22. Cherny NI, de Vries EGE, Dafni U, et al. Comparative assessment of clinical benefit using the ESMO-Magnitude of Clinical Benefit Scale version 1.1 and the ASCO Value Framework net health benefit score. J Clin Oncol 2019;37:336-349.

23. Ben-Aharon $\mathrm{O}$, Magnezi R, Leshno M, et al. Association of immunotherapy with durable survival as defined by value frameworks for cancer care. JAMA Oncol 2018;4:326-332.

24. Roydhouse JK, King-Kallimanis BL, Howie LJ, et al. Blinding and patient-reported outcome completion rates in US Food and Drug Administration cancer trial submissions, 2007-2017. J Natl Cancer Inst 2019;111:459-464.

25. Moscow JA, Fojo T, Schilsky RL. The evidence framework for precision cancer medicine. Nat Rev Clin Oncol 2018;15:183-192.

26. Zettler M, Nabhan C. Fulfillment of postmarketing requirements to the FDA for therapies granted oncology indications between 2011 and 2016. JAMA Oncol 2018;4:993-994.

27. Basch E. Toward patient-centered drug development in oncology. N Engl J Med 2013;369:397-400.

28. Allegra CJ, Goodwin PJ, Ganz PA. Can we find the positive in negative clinical trials? J Natl Cancer Inst 2019;111:637-638.

29. Nass SJ, Rothenberg ML, Pentz R, et al. Accelerating anticancer drug development-opportunities and trade-offs. Nat Rev Clin Oncol 2018;15: 777-786.

30. Gyawali B, Hey SP, Kesselheim AS. Assessment of the clinical benefit of cancer drugs receiving accelerated approval. JAMA Intern Med 2019. 179:906-913. 
Supplemental online content for:

\section{Associations With Definitive Outcomes and Clinical Benefit of Cancer Drugs at the Time of Marketing Approval and in the Postmarketing Period}

Aida Bujosa, MD; Consolación Moltó, MD; Thomas J. Hwang, AB; José Carlos Tapia, MD; Kerstin N. Vokinger, MD; Arnoud J. Templeton, MD; Ignasi Gich, MD, PhD; Agustí Barnadas, MD, PhD; Eitan Amir, MD, PhD; and Ariadna Tibau, MD, PhD

J Natl Compr Canc Netw, doi: 10.6004/jnccn.2021.7003

eTable 1: Predictors of Benefit in the Noncurative Setting 


\section{eTable 1. Predictors of Benefit in the Noncurative Setting}

Univariable Analysis

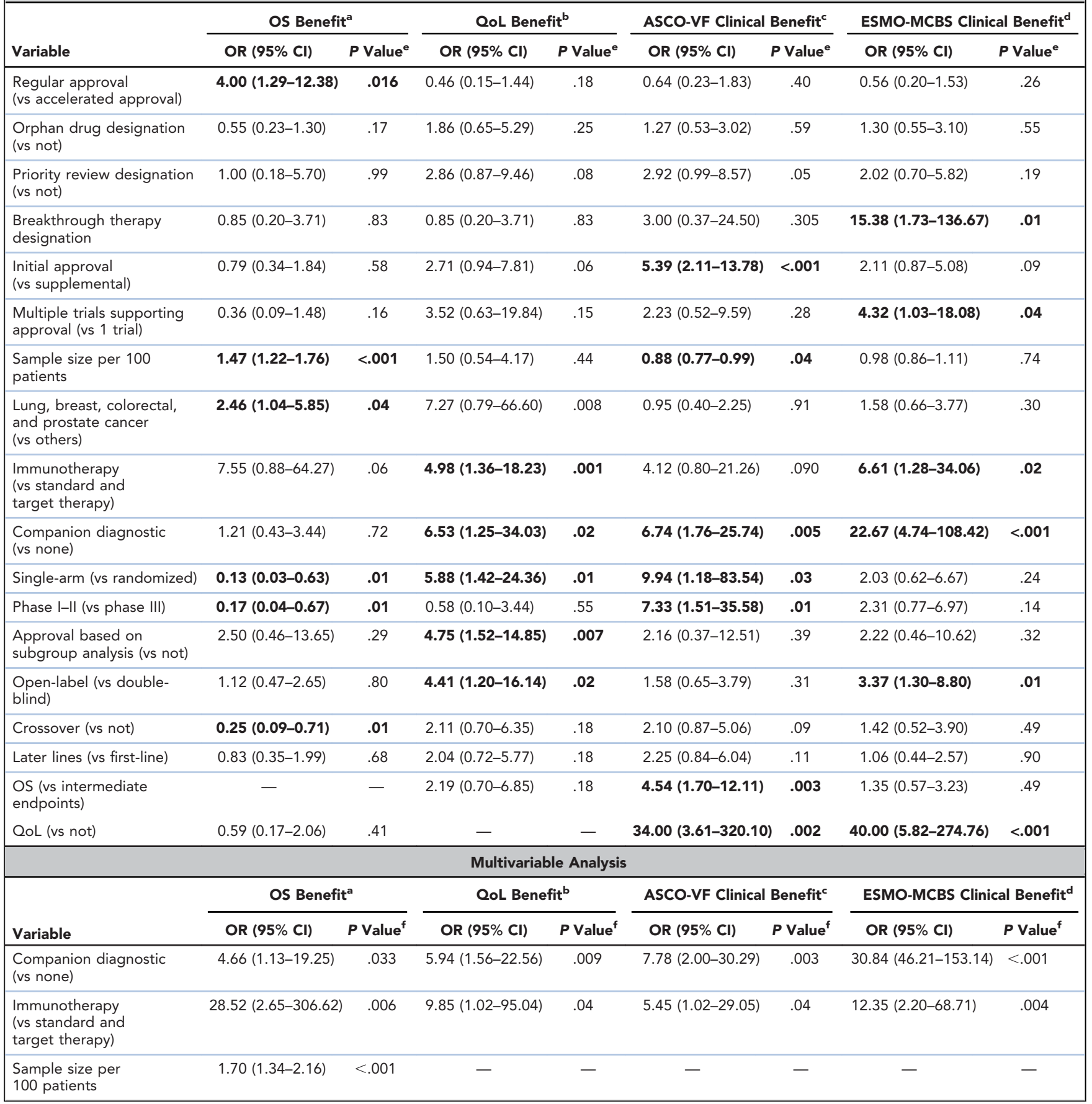

Bold indicates statistically significant $P$ value.

Abbreviations: ASCO-VF, ASCO Value Framework; ESMO-MCBS, ESMO-Magnitude of Clinical Benefit Scale; OR, odds ratio; OS, overall survival; QoL, quality of life.

${ }^{a} A$ drug was considered to have shown an OS benefit if a statistically significant benefit was observed with experimental therapy.

${ }^{\mathrm{b}} \mathrm{A}$ drug was considered to have shown a QoL benefit if a statistically significant difference was reported between the drug and experimental arm among randomized controlled trials and between baseline and after treatment in single-arm trials.

${ }^{c}$ High clinical benefit for ASCO-VF was considered a threshold score of $\geq 45$.

dHigh clinical benefit for ESMO-MCBS was considered a grade of A or B for trials of curative intent and a grade of 4 or 5 for those of palliative intent.

e Based on univariable logistic regression. All $P$ values are 2-sided.

${ }^{f}$ Multivariable models were adjusted for variables with $P$ values $<.05$ in the univariable model and showing benefit for at least 2 benefit outcomes: sample size (continuous), companion diagnostic (yes vs no), immunotherapy (yes vs no), and type of trial (single-arm vs randomized). 